\title{
Proposed Biology of the Spontaneous Remission of Cancer
}

\author{
Behzad Niakan*
}

Pharm. D, USA

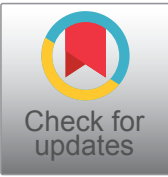

*Corresponding author: Behzad Niakan, Pharm. D., P.O. Box 25817, Los Angeles, Ca. 90025, USA, Tel: (310)-925-2720

\begin{abstract}
Two common factors were identified among the case reports of the prolonged spontaneous remission and regression of cancer. The prolonged spontaneous remissions of cancer may be preceded by a low blood oxygen delivery to the tumor and a short, steady or rapid malignant growth. The steady or rapid growth of primary tumor not the growth of new metastases.
\end{abstract}

\section{Introduction}

Case reports of the spontaneous disappearance of verified cancer without any medical intervention has baffled researcher. The disappearance of all signs and symptoms of cancer without any medical intervention. Before the 1960's case reports of spontaneous regression or remission of cancer was dismissed by the medical community as a misdiagnosis. This began to change after the publication of the book "spontaneous regression of cancer" by Dr. Everson and Dr. Cole in 1966 which referenced many of these case reports which the authors considered having sufficient documentation as being cancer. The spontaneous disappearance of cancer may occur in many different cancers. In some types of cancer more frequently. It may last for a short period or for a very prolonged period. The rate of a spontaneous regression or remission of cancer is not known. It is of interest that some of these case reports of the spontaneous remission or regressions of cancer may be commonly preceded by specific events such as infections particularly pneumonia, pulmonary edema, pulmonary metastases, blood transfusion, termination of pregnancy, biopsy, etc.

Spontaneous remission of hepatocellular carcinoma may occur more commonly after tumor hypoxia (occlusion of the hepatic artery or the portal vein).
Spontaneous remission of renal cell carcinoma may occur more commonly after nephrectomy in patients with pulmonary metastases. Spontaneous remissions of leukemia may occur more commonly after pneumonia. Spontaneous remission of Merkel cell carcinoma may occur more commonly after biopsy, etc. It is interesting that these specific events are not specific to a type of cancer, they may occur in different types of cancer. Yet, they have a higher propensity in certain types of cancer. A common denominator was sought.

The etiology of the spontaneous regression or remission of cancer is unknown. Theories regarding the pathophysiology of the spontaneous regression or remission of cancer vary. It might be attributed to an immune activation, withdrawal of a possible causative agent, hormonal change, change in blood supply, cell apoptosis, tumor necrosis, infection, cytokines, chemokines, herbal remedies, etc. this study suggest that a steady or rapid growth of primary tumor faced with a low blood oxygen may result in a spontaneous regression or remission of cancer.

\section{Methods}

Different disciplines related to cancer were studied such as biology, immunology, endocrinology, neurology, etc. as it relates to cancer. Later focus was made on the reported cases of the spontaneous regression or remission of cancer. Case Reports of the spontaneous remission and regression of cancer since the late 1800's was studied. They were classified as short and temporary or prolonged remissions. Afterwards the case reports of prolonged spontaneous remissions or regressions of cancer were classified by tissue type and events preceding the spontaneous regression or remission of cancer. The events most commonly preceding each 
type of cancer seem to be different. Yet, the events are not specific to a type of tumor. A common denominator was sought among the events preceding the prolonged spontaneous regression or remission of cancer. This study has taken over forty years.

\section{Results}

Prolonged Spontaneous remission of cancer has been reported after pneumonia [1], pulmonary edema [2], hemorrhage [3,4] low hemoglobulin [5], Tumor hypoxia (occlusion of hepatic artery or portal vein) [6], nephrectomy in the presence of pulmonary metastases [7-10], liver cirrhosis [11,12], jaundice [13,14] biopsy [1518], etc. All have in common a low blood oxygen delivery to the tumor. Pneumonia, pulmonary metastases and pulmonary edema may slow oxygen delivery through the longs. Hemorrhage and low hemoglobulin may result in low oxygen transport. Spontaneous remission of hepatocellular carcinoma often time is preceded by occlusion of hepatic artery or portal vein which blocks or slows down blood oxygen to the tumor. Spontaneous remission of renal cell carcinoma is often time preceded by nephrectomy in the presence of pulmonary metastases. The kidney produces erythropoietin which signals the bone to produce red cells. A combined low erythropoietin and pulmonary metastases may significantly reduce blood oxygen supply. Liver cirrhosis may cause microcytic anemia. Hepatic jaundice may cause anemia. Biopsy may result in breakage of a vessel and bleeding within the tumor particularly Merkel cell carcinoma. Implying low blood oxygen is implicated in the prolonged spontaneous remission of cancer.

Case reports of prolonged Spontaneous remission or regression of cancer seem to have in common a short, steady or rapid growth just prior to the spontaneous remission or regression [19]. The short, steady or rapid growth of the primary tumor not new metastases. Otherwise, the new metastases may adjust to the lower blood oxygen level.

\section{Discussion}

Low blood oxygen and a short period of steady or rapid malignant growth just prior to the prolonged spontaneous remission or regression of cancer seem to be significant. One may speculate of its significance.

Malignant tumors can grow faster when certain substrate is provided. Also, malignant tumors grow faster when the blood oxygen level is low. The combination of both may drive the tumor to grow faster than it can handle. Thereby, a dysfunctional tumor maybe removed by the immune system.

As a tumor burden increase the growth rate of the tumor may slow down. Hinting of a dynamic growth limiting mechanism. Therefore, a tumor growing steadily without slowing down may have a breakdown in tumor homeostasis.
It seems when a malignant growth faces hypoxia it may become more aggressive (progression). It seems for progression to take place the tumor growth needs to slow down or stop for a short while. Possibly the opposite might hold true, if a malignant growth is growing steadily and faces resistance and its growth does not slow down then apoptosis might set in.

A malignant tumor is combined of a well oxygenated section and of a hypoxic section. The tumor cannot be all well oxygenated because it would not be malignant. It cannot be all hypoxic otherwise there would not be a malignant tissue. One may suggest a malignant growth grows while a balance is held between the well oxygenated section and the hypoxic section.

The possible implication could be the rapid malignant growth may result in the well oxygenated section to become hypoxic faster than the malignant growth being able to adjust to it. Thereby breaking the balance between the well oxygenated section and the hypoxic section by having an excessive hypoxic section. Thereafter the tumor not being viable and being recognized by the immune system as a diseased tissue and a harmonious immune response removing the malignant growth.

Malignant tumor growth may slow down when sufficient substrate is not available and adjust to the new environment. There are instances a tumor may grow steadily in the absence of adequate substrate such as rebound tumor growth which may occur after palliative radiation therapy or after palliative surgery. The malignant tissue may grow faster than it can adjust to its environment (low blood oxygen). The malignant tissue may accumulate excessive hypoxic tissue which may throw off the balance within the tumor between the well oxygenated tissue and the hypoxic tissue. Thereafter a successful immune response may remove the malignant growth.

It is interesting to note that cancer cells have an anerobic respiration. Yet, cancer cells have an increased dependency on iron compared to normal cells. The function of anerobic respiration is production of energy without oxygen. A function of iron is oxygen transport. A possible explanation might be that malignant tumors may have a co-dependence on the presence and the absence of oxygen.

Tumor growths have anerobic respiration in the presence of oxygen. Hypoxia enhances cancerous growth. Yet too much hypoxia may accelerate tumor growth faster than the tumor could adjust to it. Therefore, a dysfunctional tumor and its removal by the immune system.

Respiration is a chemical reaction taking place in all living cells and release energy from glucose. Cellular respiration can take place in the presence of oxygen or in the absence of oxygen. 
During aerobic cellular respiration, glucose reacts with oxygen, forming ATP that can be used by the cell. During anaerobic cellular respiration, glucose is broken down without oxygen. Less energy is released but more quickly (about 100 times faster) than aerobic respiration. Lactate dehydrogenase enzyme (LDH) is an important enzyme of the anaerobic metabolism pathway. The function of the enzyme is to catalyze the reversible conversion of lactate to pyruvate and vice versa.

The reaction catalyzed by enzyme lactate dehydrogenase (LDH) may proceed in either direction, but the conversion of pyruvate to lactate is inhibited by acidic conditions.

When cells become exposed to anaerobic or hypoxic conditions, the production of ATP by oxidative phosphorylation (aerobic respiration) becomes disrupted. This process demand cells to produce energy by alternate metabolism. Consequently, lactate dehydrogenase $(\mathrm{LDH})$ is up regulated in such conditions to cater to the need for energy production. However, lactate produced during anaerobic conversion of glucose meets a dead end of metabolism. It cannot go further metabolism in any tissue except the liver. Hence lactate is Released in the blood and transported to the liver.

Cancer cells tend to use fermentation (anerobic respiration) for obtaining energy even in aerobic conditions, coining the term aerobic glycolysis. Cancer cells use glucose about 200 times more than normal cells.

In cancer cells, the function of LDH (lactate Dehydrogenase), specifically LDHA (lactate Dehydrogenase A), is modified as to compared to the normal cells. Cancer cells employ LDH to increase their aerobic metabolism (glycolysis and ATP production, and lactate production) even in the presence of oxygen. This process is known as the Warburg effect.

Possibly a primary tumor growing steadily and rapidly could be producing lactic acid faster than it could be excreted from the primary tumor. Resulting within the tumor becoming more acidic and inactivating the enzyme lactate dehydrogenase. In addition, hypoxic tissues become acidic. Possibly the low blood oxygen delivered to a rapidly growing primary tumor may have further caused within the tumor becoming more acidic and further inhibition of the lactate dehydrogenase enzyme. Possibly the inactivation of the lactate dehydrogenase enzyme within the primary tumor has resulted in a dysfunctional tumor and its removal by the immune system. Possibly once, the primary tumor is removed by the immune system, immunity is developed and all cancer cells are removed by the immune system.

A loop seems to be occurring if a primary tumor is growing steadily or rapidly. As a primary tumor grows steadily or rapidly more lactic acid is produced. Resulting within the tumor becoming more acidic. Resulting in the inactivation of the enzyme lactate dehydrogenase. Resulting in a lack of energy for the primary tumor and its breakdown. A primary tumor growing steadily or rapidly faced with hypoglycemia possibly may remit spontaneously. Since, the suggested loop becomes exacerbated due to lack of glucose.

It is noteworthy that spontaneous remission of cancer in mice or rat may occur if the tumor grows steadily and faced by a low blood oxygen level.

1. The paper published by C. A. Apfel, et al. [20] indicated that a serial evacuation of the ascites fluid of mice with transplantable mouse ascites tumors resulted in a large ascites fluid which was followed by a spontaneous remission. Serial evacuation of ascites fluid was each time replaced by a larger ascites fluid (steady growth) until it reached a large size and followed by a spontaneous remission. Also, large ascites fluid can increase pressure on the diaphragm causing pleural effusions causing a lower oxygen delivery by lungs. Therefore, a steady tumor growth faced with a low blood oxygen. It is interesting to note, that replacing the cell-free ascitic fluid had the opposite effect.

2. The paper published by Jana Kovalska, et al. [21]. Indicated that Lewis Rat Sacroma Model in rats had a continuous growth followed by a spontaneous regression. The authors noted that during the progressive growth and spontaneous regression that followed afterwards there was a decrease in the number of red blood cells, hemoglobin and hematocrit. Possibly the steady tumor growth resulted in an increase in blood volume due to a larger tumor burden. Yet, the lungs delivered the same amount of oxygen. Therefore, a decrease in blood oxygen level. The point made is that a steady rapid tumor growth in the presence of low blood oxygen possibly resulted in the regression or remission. Otherwise, a slow or intermittent tumor growth may adjust to a lower blood oxygen level.

3. The paper published by Manon Buijs, et al. [22]. Indicated that N1S1 cells and McA-RH7777 cells were implanted in the liver of Sprague-Dawley rats. Tumor growth continued to a size and afterwards regressed and complete regression of all tumors were seen five to six weeks after tumor implantation. According to the authors tumors grew to relatively large size compared to the overall size of the liver in rats. Possibly the increase in tumor size has resulted in an increase in blood volume and the lungs delivering the same amount of oxygen has resulted in a drop in blood oxygen level. Also, liver tumor may 
concentrate in iron and reduce blood oxygen delivery from lungs. Therefore, a steady growth of a tumor faced with an increasing lower blood oxygen level.

Proposed biological mechanism of the spontaneous remission of cancer. Spontaneous remissions of cancer may be preceded by two events. A brief steady or rapid growth and a low oxygen delivery to the tumor tissue. Cancerous cells have anaerobic respiration with the end by product of lactic acid. When a malignant tissue grows steadily or rapidly it could produce lactic acid faster than its clearance. Therefore, a buildup of lactic acid in the malignant tissue. Furthermore, low oxygenation of malignant tissue could further push the production of lactic acid. Resulting in a very acidic malignant tissue or acidosis within the malignant tissue. Lactate dehydrogenase is an important enzyme in the anaerobic pathway. The activity of enzyme lactate dehydrogenase becomes inhibited during high acidic conditions (substrate inhibition). With the inactivity of the enzyme lactate dehydrogenase the malignant tissue and its microenvironment breaks down. Once, the malignant tissue becomes dysfunctional it becomes removed by the immune system. Afterwards immunity develops against the malignant growth and residual cancer cells are removed.

\section{POSSIBLE EXPERIMENTALTREATMENTFOR A CANCER} IN WHICH THE PRIMARY TUMOR IS ENCAPUSLATED WHETHER SPREAD OR NOT

1) Injecting lactic acid into a primary tumor of an aggressively growing malignant tumor such as pancreatic cancer to induce high acidic environment within the primary tumor. The purpose is for the tumor to retain lactic acid faster than it releases lactic acid. Resulting in a high acidic environment which may inactivate the enzyme lactate dehydrogenase. An encapsulated malignant tumor would be ideal. MRI imaging can detect presence of a tumor capsule

2) Injecting iron chelators or angiogenesis inhibitors or anything else within a primary tumor of an aggressively growing malignancy such as hepatocellular carcinoma to rapidly reduce oxygen within a primary tumor and to induce a high acidic environment. Encapsulated hepatocellular carcinoma would be preferable

3) Using needle biopsy to rapidly remove blood (or interstitial tissue or both) from the vascularization of a primary tumor of an aggressively growing malignancy such as Merkel cell carcinoma. Encapsulated Merkel cell carcinoma would be preferable

4) low dose radiation to partially inactivate a primary tumor of an aggressively growing malignancy such as renal cell carcinoma and following it with injecting the primary tumor with lactic acid or iron chelators or angiogenesis inhibitors or a combination to induce a hyper-acidic environment within the tumor
5) Using hyperthermia to lower the PH of a primary cancerous growth and following it with injection of lactic acid or iron chelators or angiogenesis inhibitors into the heated cancerous tissue. Encapsulated growth is preferable. The effect of hyperthermia on the tumor capsule is of consideration

6) Inducing acidosis within an encapsulated cancerous growth

\section{Caution}

A hypothesis has been suggested without any experimental proof for its correctness. Experimental treatment requires medical supervision. Oxygen levels may drop too low, Tumor growth may affect a vital organ or cause irreversible damage. Inducing tumor growth may result in tumor progression (more aggressive tumors), etc.

\section{Disclaimer}

Always seek the advice of your physician or other qualified health care provider with any questions you may have regarding a medical condition. Never disregard professional medical advice or delay in seeking it because you have read this study. This is not intended to be a substitute for medical advice, diagnosis, or treatment in regard to any patient.

\section{References}

1. Rashidi A, Fisher S (2015) Spontaneous remission of acute myeloid leukemia. Leuk Lymphoma 56: 1727-1734.

2. Chin KM, Chan CY, Lee SY (2018) Spontaneous regression of pancreatic cancer: A case report and literature review. Int J Surg Case Rep 42: 55-59.

3. Chang WY (2000) Complete spontaneous regression of cancer: four case reports, review of the literature, and discussion of possible mechanism involved. Hawaii Med $\mathrm{J}$ 59: 379-387.

4. Tocci G, Conte A, Guarascio P, Visco G (1990) Spontaneous remission of hepatocellular carcinoma after massive gastrointestinal bleeding. BMJ 300: 641-642.

5. Mozafari R, Moeinian M, Asadollahi-Amin A (2017) Spontaneous complete remission in a patient with acute myeloid leukemia and severe sepsis. Case Rep Hematol 2017: 9593750.

6. Huz JI, Melis M, Sarpel U (2012) Spontaneous regression of hepatocellular carcinoma is most often associated with tumor hypoxia or a systemic inflammatory response. HPB(oxford) 14: 500-505.

7. Vizel M, Oster MW, Austin JH (1979) Spontaneous regression of pulmonary metastasis after nephrectomy for renal cell carcinoma. J Surg Oncol 12: 175-180.

8. Nakano E, Sonoda T, Fujioka H, Okuyama A, Matsuda $M$, et al. (1984) Spontaneous regression of pulmonary metastases after nephrectomy for renal cell carcinoma Eur Urol 10: 212-213.

9. Thoroddsen A, Gudbjartsson T, Geirsson G, Agnarsson BA, Magnusson K (2002) Spontaneous regression of pleural metastases after nephrectomy for renal cell carcinoma - A histologically verified case with nine-year follow up. Scand J Urol 36: 396-398. 
10. Skriver M, Nielsen JK (1995) Spontaneous regression of pulmonary metastases after nephrectomy for renal cell carcinoma. Ugeskr Laeger 157: 6138-6139.

11. Kondo S, Okusaka T, Ueno H, Ikeda M, Morizane C (2006) Spontaneous regression of hepatocellular carcinoma. Int $\mathrm{J}$ Clin Oncol 11: 407-411.

12. Suzuki M, Okazaki N, Yoshino M, Yoshida T (1989) Spontaneous regression of hepatocellular carcinoma-a case report. Hepatogastroenterology 36: 160-163.

13. Ken min chin, Chung yip chang, Ser yee lee (1982) Spontaneous regression of hepatocellular carcinoma. Gastroenterology 82: 770-774.

14. Gottfried EB, Steller R, Paronetto F, Lieber CS (1982) Spontaneous regression of hepatocellular carcinoma. Gastroenterology 82: 770-774.

15. Maruo K, Kayashima KI, Ono T (2000) Regressing merkel cell carcinoma - a case showing replacement of tumor cells by foamy cells. Br J Dermatol 142: 1184-1189.

16. Branch S, et al. (2018) Spontaneous regression of merkel cell carcinoma. Cutis 101: 301-305.
17. Pang C, Sharma D, Sankar T (2015) Spontaneous regression of merkel cell carcinoma: $A$ case report and review of the literature. Int J Surg Case Rep 7: 104-108.

18. Terui H, Fujimura T, Kakizaki A, Furudate S, Aiba S (2016) Merkel cell carcinoma with spontaneous regression: a case report and immunohistochemical study. Case Rep Dermatol 8: 52-58.

19. Niakan B (1993) Steady tumor growth and spontaneous disappearance of cancer. J Natl Cancer Inst 85: 68-69.

20. Apffel CA, Arnason BG, Twinam CW Harris CA (1966) Recovery with immunity after serial tapping of transplantable mouse ascites tumours. Br J Cancer 20: 122-126.

21. Kovalská J, Mishra R, Jebavý L, Makovický P, Janda J, et al. (2015) Tumor progression and spontaneous regression in the lewis rat sarcoma model. Anticancer Res 35: 65396549.

22. Buijs M, Geschwind JFH, Syed LH, Ganapathy-Kanniappan S, Kunjithapatham R, et al. (2012) Spontaneous regression in a syngeneic rat model of liver cancer: implications for survival studies. J Vasc Interv Radiol 23: 1685-1691. 